\title{
SOBRE EL VENEZOLANISMO ARROCHELADO
}

\author{
ON THE VENEZUELAN WORD ARROCHELADO
}

\author{
EnRIQue Obediente Sosa \\ Universidad de los Andes (Mérida, Venezuela) \\ Academia Venezolana de la Lengua \\ enriobe@gmail.com
}

RESUMEN: En la presente nota se reflexiona sobre el vocablo arrochelado y se aportan algunas novedades y precisiones sobre su origen, particularmente sobre la datación de sus distintas acepciones. Se presentarán los diversos significados que tiene o ha tenido a lo largo del tiempo, así como la extensión diatópica de la palabra. Para ello, se partirá de lo ya expuesto magistralmente por el gran filólogo Ángel Rosenblat, que se ampliará con lo que revelan nuevas fuentes documentales y los corpus digitales que han venido a enriquecer el conocimiento del léxico español en sus distintas variedades.

Palabras clave: lexicografía; venezolanismos; arrochelado; rochela; documentación colonial.

Abstract: The present note reflects on the term arrochelado, contributing information or details about its origin and, in particular, about the dating of its different meanings. The different sense that the term has acquired over time will be presented as well as the diatopic extension of the word. In order to achieve this, we will begin by resuming what has already been masterfully expounded by the great philologist Ángel Rosenblat, and then expand on this offering new documentary sources and digital corpora which have subsequently enriched our knowledge of the Spanish lexicon in its different varieties.

Keywords: lexicography; venezuelanisms; arrochelado; rochela; colonial documentation.

Recepción: 11 de junio de 2018; aceptación: 3 de septiembre de 2018.

D.R. (C) 2019. Nueva Revista de Filología Hispánica Licencia Creative Commons Attribution-NonCommercial (CC BY-NC) 4.0 International 
El punto de partida de este renovado interés por la palabra arrochelado lo constituyó el haberla encontrado recientemente en un texto de hace más de dos siglos, escrito en la Mérida venezolana. En efecto, la lectura y transcripción de un legajo merideño del siglo XVIII relativo a un caso de estupro nos permitió reencontrarnos con la palabra en cuestión, palabra ciertamente ya poco empleada en el español venezolano, como tendremos ocasión de ver, pero bastante frecuente entre mediados del siglo XVIII y principios del XX.

Consideramos que no estaría de más presentar el vocablo en el contexto del legajo mencionado, del cual se dará en seguida un breve resumen. El conjunto de los documentos originales que constituye el legajo reposa en el Archivo General del Estado Mérida (AGEM) bajo la signatura Estupro, tomo 1, ff. 5r-15r ${ }^{1}$.

El 17 de mayo de 1786, Xavier de Lobo introduce un escrito de querella contra Francisco Ramírez, a quien acusa de haber seducido a su hija menor Rafaela, la cual quedó "gravidada ${ }^{2}$ y del todo perdida" (f. 5r), razón por la cual solicita que el mencionado Ramírez sea preso, secuestrados sus bienes y desterrado de Mérida. Dada la gravedad de la acusación, el alcalde ordinario exige, entonces, la detención y embargo de bienes del joven, pero "Indagado por sus Vienes no se le Encontraron Ningunos" (f. 6v). Ramírez se defiende y rechaza la acusación de Lobo argumentando que él y Rafaela habían convenido en casarse, pero que ésta, bajo la presión y amenazas de su padre, se resistió a cumplir con su palabra. Dado, pues, que fue ella la que se retractó de lo acordado, Ramírez se considera libre de toda responsabilidad y pide al alcalde lo declare "por absuelto de tan injusta querella y dar al despresio cualesquiera persuasibas de la contraria parte mandándole poner silencio en el asunto" (f. 9r). Vistas la no comparecencia del querellante a las citaciones del tribunal y las alegaciones presentadas por Ramírez, el alcalde, que actúa como juez de la causa, lo declara libre el 3 de octubre del mismo año y absuelto de la demanda incoada por Xavier de Lobo; pero como el hecho mismo de haber embarazado a Rafaela "Nunca les dejó de Ser Perjudicial a Ésta, y a sus Padres, tanto en el honor de todos como en haver ynabilitado la Muchacha para Poderse matrimoniar fácilmente con otro" (f. 14r), le impuso como castigo, además de la prisión ya sufrida, el pago de las costas procesales.

En el escrito de querella presentado por el padre de Rafaela se lee que Ramírez -según Xavier de Lobo- se había "mantenido de poco tiempo acá arrochelado en la vesindad de mi casa mientras mi muger se hallaba en cama grabemente enferma, y introduciéndose

${ }^{1}$ El legajo está actualmente disponible en el CORDIAM (Venezuela, año 1786, jurídico). Se mantiene en las citas la transcripción paleográfica del texto, pero se moderniza acentuación. En cursiva, desato las abreviaturas.

${ }^{2}$ Por grávida 'preñada'. 
de noche furtibamente ha comunicado y comerciado con la dicha mi hija, de que ha resultado ésta gravidada" (f. $5 \mathrm{r})^{3}$. A lo que replica Ramírez, en su carta de impugnación de la acusación de estupro, en los siguientes términos: "aunque asevera el Lobo, que yo me he mantenido arrochelado en su vencindad, es en el todo falsa su Depocición... antes bien teniendo, mi casa en aquel vesindario he procurado evitar el paso de su calle ext[r] aviando otras para ir a mi casa" (f. 8v).

Así pues, mientras Lobo afirma que el enamorado de su hija estaba arrochelado en la vecindad de su casa, éste lo niega y precisa que, a pesar de tener su propia casa en el mismo vecindario que Rafaela, procuraba evitar pasar por la calle donde vivían los Lobo, tomando otras para llegar a la suya.

¿Qué significa arrochelado en el contexto de este documento?

\section{LAS DIVERSAS ROCHELAS}

Vayamos en primer lugar al artículo que Ángel Rosenblat dedica a la voz rochela (raíz de arrochelado) en Buenas y malas palabras ${ }^{4}$, antes de examinar -como exige todo trabajo de carácter lexicográfico- lo que corpus y repertorios léxicos disponibles dicen al respecto ${ }^{5}$. El maestro Rosenblat considera que para comprender el significado y uso de la palabra rochela hay que remontarse necesariamente a la ciudad francesa de La Rochelle, puerto de la fachada atlántica del país galo. Rosenblat, después de pasearse por la convulsa historia de tan afamada ciudad (historia de corsarios, hugonotes e invasiones), reafirma el hecho de que, para la España de los siglos XVI y XVII, La Rochela era el lugar por antonomasia de bandolerismo, pillaje, desorden y confusión:

Así se le aparecía la ciudad protestante, refugio de corsarios, a la católica España. Es la imagen que pasó a América. Se olvidó en el resto del mundo hispánico, pero se recuerda en Venezuela, Colombia y el Ecuador, fieles a un uso que fue sin duda general entre gentes de mar (1974, t. 2, pp. 122-123).

Si así son las cosas, rochela debió de haber tenido como primer significado 'lugar de desorden', en el más amplio y extenso sentido; sin embargo, los primeros testimonios que da el Corpus diacrónico del español (CORDE) no apoyan esta afirmación. En efecto, aparte de los

${ }^{3}$ Énfasis nuestro en esta cita y la siguiente.

${ }^{4}$ Seguimos la $4^{a}$ edición publicada por Edime en 4 tomos, correspondiente a 1974. Rochela está en el tomo 2, pp. 118-125. Recuérdese que la $1^{\text {a }}$ edición data de 1956.

${ }^{5}$ Véase la lista de unos y otros al final de este trabajo en Referencias. 
primeros cuatro testimonios de finales del siglo xv, en los que Rochela es topónimo, los siguientes que muestra el corpus significan 'refugio' (s. XVII), 'algazara' (s. XIX) y 'reunión de ganado salvaje' (s. XX), en ese orden, tal como se puede apreciar en las citas que copiamos abajo:

...tampoco se quisieron diuertir mucho los Españoles, mirando a ocupar presto su rochela; y assí endereçaron a sus fortificaciones, y las ocuparon con sus pieças, municiones, y armas (1667, Francisco Combés, Historia de Mindanao y Joló, Filipinas).

Y lo último se infiere ser assí, como dixe en el libro primero, que todas o las más naciones son de gente fugitiva, que no por comodidades de aquellos montes, sino por su retiro los abitaron, siendo la rochela escondida que buscaron para gozar del ocio apetecido de su natural y por mantenerse en las costumbres bárbaras de su gentilismo (1684, Manuel Rodríguez, El Marañón y Amazonas. Historia de los descubrimientos, Ecuador).

-¡Ave María, Agusto! -exclamó Mina, entrando-. ¡Me admiro de que haya podido dormir con la rochela que tienen aquellas sinvergüenzas! Me fui a asomar a ver qué era y ai s'están riendo de todo el que pasa. ¡Óigalas! (1896, Tomás Carrasquilla, Frutos de mi tierra, Colombia).

...diciéndoles: "Ensillen ligero y salgan ahora mismo. En las sabanas de Lagartijera está una rochela de cimarrones. Son setenta y cinco reses y todas van a caer suavecitas" (1929, Rómulo Gallegos, Doña Bárbara, Venezuela).

El Corpus del Nuevo diccionario histórico (CDH), sin embargo, trae un testimonio anterior, ya anotado por Rosenblat y considerado como primera documentación de rochela por Corominas y Pascual (19801991, s.v. roca), y éste sí con el valor de 'lugar de desorden y confusión'. Se trata del uso metonímico del topónimo francés que se lee en el Guzmán de Alfarache (1604) de Mateo Alemán (Segunda parte, libro 2, cap. 4, apud Rosenblat 1974, t. 2, p. 122): "En resolución, todo el mundo es la Rochela en este caso, cada cual vive para sí, quien pilla y sólo pagan los desdichados como tú”. Es decir, el mundo, todo el mundo, es un lugar de desorden, confusión y pillaje. La Rochela tiene, pues, en ese texto, un sentido extendido, que podría considerarse como la transición entre el nombre propio y el común polisémico a que aquél daría lugar.

Llama la atención que el Diccionario enciclopédico de la lengua castellana de Elías Zerolo et al. de 1895 tenga la siguiente entrada: "ROCHELA (LA). Todo el mundo es la Rochela. ref. En todas partes cuecen habas. «En resolución todo el mundo es la Rochela en este caso» (Mat. Alem.)”. Este diccionario presenta, como se ve, la frase del Guzmán como un refrán equivalente a "En todas partes cuecen habas", que, según el 
Diccionario de la lengua española (DLE 2014), es expresión usada para "significar que cierto inconveniente no es exclusivo del sitio o persona de que se trata". En nuestro caso, el desorden no es pues exclusivo del tan difamado puerto francés.

El significado de rochela como 'lugar de refugio, guarida', que remite, sin duda, también a La Rochela como lugar que fue de amparo de corsarios y calvinistas, y que se corresponde de manera general con los dos primeros testimonios dados por el CORDE (el texto filipino de 1667 y el ecuatoriano de 1684), lo vamos a encontrar en textos venezolanos del siglo XVIII referido a negros, indios, zambos y mulatos huidos a los montes, donde, lejos de los centros poblados y de las misiones, podían vivir a sus anchas, en una relativa libertad que incluía frecuentemente la salida a los alrededores para cometer robos, pillajes y asaltos a casas, haciendas y personas. El primero de esos testimonios es, según reporta Rosenblat $(1974$, t. 2, p. 123), de 1776, año en que "el Prefecto de las Misiones comunicaba al Gobernador que en el Cunaviche había «una rochela de zambos»".

Hay un texto venezolano escrito en la localidad de Calabozo (en el actual estado Guárico) y alojado en el Corpus diacrónico y diatópico del Español de América (CORDIAM) en el que rochela equivale a 'refugio', mas no se refiere al de fugitivos escapados al monte, sino específicamente al de maleantes. En dicho documento, efectivamente, se habla de unos "vagantes" que encuentran amparo en casa de un particular: “...ha sabido por barios sujetos que la casa de aquél es rochela de bagantes, y que asimismo es casa donde llegan barios tratantes con sus mercancías, y éstos espenden hallí sus efectos" (Venezuela, año 1793, jurídico, $\operatorname{CORDIAM}^{6}$ ). La rochela es entonces guarida y refugio de cualquier calaña de individuos que se esconden o guarecen por una u otra razón.

De la acepción referida a personas fugitivas, se pasó, de acuerdo con Ángel Rosenblat, a "reunión de ganado salvaje e inquieto" (1974, t. 2, p. 119) y "lugar donde se reúne o refugia el ganado cimarrón, donde encuentra agua y pasto" (p. 120). Y cita un testimonio de 1799 recogido por Lisandro Alvarado según el cual el prefecto de las misiones de Guayana informa sobre la misión de San Miguel del Palmar, fundada en una loma de sabana, pero "del todo perdida por el motivo de los espineros, crobales y chiribitales, que son todos rochelas y cuevas de tigres" (id.). Hemos encontrado, sin embargo, un texto de fecha anterior, de 1758, escrito en la población de Güigüe (en el actual estado Carabobo), en el que se lee que a un testigo, declarando en contra de un acusado por diversos delitos, le consta "lo que a hejecutado en el Sitio del pao En conjunta de Don felis sárate. manteniendo rrochelas, y llebando mulas, que Vrtaban poniéndolas en Vn

${ }^{6}$ Único ejemplo de rochela como sustantivo dado por este corpus; los otros dos son topónimos. 
potrero oculto en dicho valle" (Archivo General de la Nación, Capitanía General "Diversos", tomo XXXIV, N 5, f. 9r [en Medina Ceballos 2014, doc. 1]). Aquí estamos en presencia de rochela como lugar donde se reúnen animales, pero más que a su refugio espontáneo, la palabra remite a 'escondite de bestias hurtadas'. Este testimonio sería, por tanto, el primero en el que la voz rochela hace referencia a 'reunión de animales' o 'lugar en que se juntan', adelantándose en casi dos siglos al que recoge el CORDE, el de 1929 tomado de un texto galleguiano.

En cuanto a la acepción 'bullicio, algazara' -que bastante debió de haber habido no sólo en La Rochela, sino en toda "rochela humana", porque el desorden y la confusión se acompañan generalmente de ruido-, debemos hacer notar que a partir de ella han surgido otras relacionadas semánticamente en las que están presentes las ideas de 'diversión', 'travesura', acepciones referidas tanto a comportamientos infantiles como de adultos (cf. supra el ejemplo colombiano de 1896), y que llegan incluso a connotar 'amorío', tanto en su valor de 'relación amorosa superficial y pasajera' como de 'enamoramiento'. La primera documentación de esta última acepción se remonta a 1929, según datos del CDH, en texto venezolano: "Ya me dijeron también que tienes una rochelita con una de las muchachas de Paso Real. Sé que has estado allá poniendo joropos y empatando las noches con las noches en una sola parranda" (R. Gallegos, Doña Bárbara).

El orden cronológico de las diversas acepciones de rochela sería, entonces, de acuerdo con la documentación disponible hasta el momento, el siguiente: 'lugar de desorden, confusión y pillaje' (finales del s. XVI), 'reunión y refugio de personas, particularmente de fugitivos y malvivientes' (s. XVII) ${ }^{7}$, 'lugar que sirve de escondite de ganado robado', 'reunión de ganado salvaje', 'lugar frecuentado por ese ganado' (s. XVIII), 'bullicio, algazara' (s. XIX), 'amorío' (s. XX).

Pasemos inmediatamente a examinar lo que traen los repertorios lexicográficos consultados. El primer diccionario que recoge la palabra rochela como sustantivo común es el Diccionario de la lengua española de José Alemany y Bolufer de 1917: "f. Amér. En Colombia y Venezuela, gran ruido, algazara”. Es también el primero que da entrada a su derivado rochelear: “Amér. En Venezuela, retozar, juguetear”. Hay que esperar el diccionario académico de 1985 (DRAE) para encontrar las acepciones referidas al ganado: "Venez. Reunión de ganado inquieto. Por ext., lugar donde se reúne este ganado", acepciones que elimina en la edición de 1992 para reintroducirlas, junto con el verbo rochelear, en la de 2001 y repetir, con ligerísima modificación, en el Diccionario de la lengua española (DLE) de 2014: "rochela 1. f. Col. y Ven.

${ }^{7}$ No descartamos la probabilidad de que el empleo de rochela con el significado de 'refugio' haya precedido al de 'lugar de desorden', pero la documentación existente no permite afirmarlo. 
Bullicio, algazara. 2. f. Ven. Hábito de algunos animales de frecuentar un lugar. 3. f. Ven. Lugar frecuentado por algunos animales", "rochelear 1. intr. coloq. Ven. Formar o hacer rochela. 2. intr. coloq. Ven. retozar (|| saltar y corretear) ${ }^{8}$. 3. intr. coloq. Ven. Bromear, tomar el pelo".

El Diccionario de americanismos (DAMER 2010) de la Asociación de Academias de la Lengua Española (ASALE) da, por su parte, más información sobre el término rochela:

I. 1. f. Ve. Hábito de algunos animales de frecuentar un lugar.

2. Ve. Conjunto de ganado salvaje o cimarrón.

3. Ve. Lugar frecuentado por algunos animales, generalmente por ser el sitio en el que se han criado.

II. 1. f. Co. Ve. Desorden y bullicio festivo que hace un grupo de personas. pop.

Igualmente, sobre el derivado rochelear:

I. intr. Co, Ve. Formar o hacer rochela, alboroto o bullicio. pop.

II. intr. Ve. Andar una persona o un animal inquieto o revoltoso de una parte a otra.

III. intr. Ve. Bromear, tomar el pelo.

Hay pocas diferencias entre lo señalado por el $D L E$ y por el $D A M E R$; llama la atención que el $D L E$ no registre 'conjunto de ganado salvaje o cimarrón', una de las acepciones más frecuentes de rochela. Del mismo modo, es significativo que sólo el $D A M E R$, de entre todos los repertorios consultados, registre la acepción verbal 'andar una persona o un animal inquieto o revoltoso de una parte a otra', acepción de la que no hemos encontrado ni otro registro ni testimonio alguno.

\section{DE ROCHELA A ARROCHELARSE}

Visto y comentado lo que sobre rochela nos enseñan Rosenblat y los corpus y repertorios lexicográficos consultados, tiempo es de ocuparnos de las formas derivadas arrochelarse y arrochelado. Rosenblat afirma al respecto que a partir del momento en que la rochela se transformó en la querencia ${ }^{9}$, se formó arrochelarse con el significado de

${ }^{8}$ En la edición de 2001, "Ven. retozar (|| travesear)".

9 Interpretando a Rosenblat, debió de haber habido el siguiente movimiento semántico: rochela = 'lugar de reunión o refugio del ganado donde encuentra agua y pasto' > 'lugar en el que se siente a gusto' > 'lugar del que no se quiere ir', es decir 'querencia'. 
"aquerenciarse, quedarse en un lugar sin quererse marchar" (1974, t. 2, p. 120).

El CORDE registra tres casos de arrochelarse: dos de 1929 procedentes de Doña Bárbara de Gallegos: "impedía que la hacienda altamireña pasase a arrochelarse en los lambederos de la finca vecina” y "para impedir que el ganado pase a arrochelarse en los lambederos de La Barquereña”. Ambos ejemplos hacen referencia al gusto del ganado por ir y estar en los lugares salitrosos de otras propiedades. El tercer ejemplo, bastante anterior (del siglo XVIII), ostenta el significado de 'refugiarse forajidos':

ay en este valle vagamundos y algunos pícaros rrateros que se arrochelan en vn paraje que llaman Palma por no hauer Cabo ni ninguno que los persiga en el Valle al que acuden por la vecindad al Puerto: sus Naturales son desidiosos por lo común (1765, Juan de Salas, Ciudad de Nirgua. [Relaciones geográficas de la gobernación de Venezuela]).

Arrochelarse, por lo visto en los ejemplos arriba citados, se aplicó en primer lugar a personas y, sólo más tarde, a animales.

El CDH trae los mismos ejemplos del CORDE, más otro de arrochelado tomado de un texto colombiano de historia que vale la pena citar:

Quienes encabezaron este movimiento fueron sobre todo mestizos y poblaciones marginales, que buscaron acomodo en los nuevos asentamientos... La Corona al observar esta tendencia, incrementó también las fundaciones, con el objeto de evitar que las gentes se establecieran "arrocheladas", como se decía a la colonización espontánea, acostumbrándose a vivir "sin Dios ni ley". Así, las autoridades coloniales buscaron que las gentes vivieran "en policía", dentro de los controles sociales y morales que debían regir la vida urbana (1992, Fabio Puyo, Bogotá) ${ }^{10}$.

Allí es evidente el significado de 'establecerse personas en un lugar de manera más o menos desordenada'. Nótese que el texto da a entender que arrochelado ya era, para finales del siglo xx, un término en desuso en Colombia al señalar "como se decía”, en un contexto que remite a la época de la colonia, específicamente a finales del siglo XVIII.

En el CORDIAM no hay ejemplos de derivados de rochela, excepto los dos casos de arrochelado de nuestro documento merideño.

Examinados los corpus digitales, pasemos ahora a revisar los repertorios lexicográficos. El primer diccionario que registra arrochelarse es el de Alemany y Bolufer de 1917: “Amér. En Venezuela,

${ }^{10}$ Por el año de publicación del texto (1992), este ejemplo no podía aparecer en el CORDE, cuyo límite temporal superior es 1974. 
plantarse las caballerías. / Amér. En Colombia, alborotarse los caballos". La entrada y las acepciones de nuestro verbo se recogen en el diccionario académico de 1927 copiando prácticamente a Alemany y Bolufer: "Colomb. Alborotarse el caballo. / Venez. Aquerenciarse, plantarse el caballo" (DRAE 1927).

Consultado el Mapa de diccionarios académicos de la RAE (www.rae. es), constatamos que las ediciones de 1992 y de 2001 no traen ni arrochelarse ni arrochelado, como tampoco el DLE de 2014. De hecho, la última edición del diccionario académico que recoge arrochelarse (mas no arrochelado) es la de 1989, con las mismas dos acepciones que ya registraba la edición de 1927.

El Diccionario de americanismos (DAMER), por el contrario, sí registra uno y otro término con distintas acepciones cada uno, y todo referido únicamente a Venezuela:

\section{arrochelarse.}

I. 1. intr. prnl. Ve. Habituarse una persona o un animal a permanecer en un lugar sin querer irse.

2. Ve. Detenerse un animal en un lugar resistiéndose a proseguir la marcha. rur.

II. 1. intr. prnl. Ve. Juntarse varias personas para crear desorden y bullicio.

\section{arrochelado, -a.}

I. 1. adj. Ve. Referido al ganado, habituado a permanecer en la rochela, lugar.

2. Ve. Referido a persona, que frecuenta muy asiduamente un determinado lugar.

Puede observarse que la acepción I, 2 del verbo se corresponde con la que da para Venezuela Alemany y Bolufer (1917) y que más tarde reproduciría la Academia. Respecto a arrochelado, el DAMER sí trae la acepción que particularmente nos interesa, la referida a persona, y cuadra a la perfección con lo que quiere significar la palabra en el contexto del documento merideño, como veremos luego.

Pasemos ahora a examinar lo que dicen los dos principales repertorios lexicográficos del español hablado en Venezuela. Según el Diccionario del habla actual de Venezuela (DHAV) de Núñez y Pérez (1994), arrochelarse tiene cinco acepciones:

1 coloq Reunirse un grupo de personas para crear desorden, bullicio o confusión.

2 coloq Hacerse compañero frecuente de una persona.

3 coloq desp Aficionarse a un lugar que se visita con frecuencia.

4 rur Dispersarse una manada de ganado tomando distintas direcciones. 5 rur Detenerse una bestia en un lugar de un camino y no querer seguir adelante, por temor o por costumbre. 
De arrochelado, sólo dice que es un término coloquial, de uso en la región andina (estados Táchira, Mérida y Trujillo), que se aplica a la persona que "tiene un carácter obstinado o terco". Llama la atención el significado que da este diccionario de dicha voz, único, por demás, en traerla, y además con la marca diatópica Andes. Hasta donde hemos podido averiguar, ninguna otra fuente registra el vocablo con el valor de 'persona terca'.

Es cierto que el $D H A V$ recoge, como se acaba de ver, el verbo arrochelarse, de donde puede inferirse que arrochelado (como forma nominal que es de ese verbo) podría remitir también a cualquiera de aquellas cinco acepciones. No deja de resultar curioso que este diccionario no especifique si la $3^{\mathrm{a}}$ acepción se aplica a personas o a animales, o a ambos $^{11}$.

Veamos ahora la información que aporta Tejera (1993) en el Diccionario de venezolanismos (DIVE). La entrada arrochelado remite a arrochelarse en sus dos acepciones, a saber, " 1 . Habituarse un animal o una persona a permanecer en un lugar sin querer irse. 2. rur. Detenerse una bestia en un lugar resistiéndose a proseguir la marcha". Pero agrega un tercer significado a arrochelado: "adj hist Se decía de los esclavos fugitivos escondidos en algún lugar", que respalda con un testimonio de 1817: "Se pagarán veinticinco pesos por el esclavo que se encuentre en la cumbre o arrochelado en el monte" (Gaceta de Caracas, 7 de mayo, VI, No 130, p. 1016). Llama la atención que arrochelado $^{1}$ y arrochelado ${ }^{2}$ estén marcados por el DIVE como participios pasados, mas no arrochelado $^{3}$, que marca sólo como adjetivo, lo cual es, sin embargo, coherente, pues para este diccionario arrochelarse no tiene como acepción 'refugiarse los esclavos fugitivos'.

De arrochelado ${ }^{1}$, el DIVE presenta como testimonio el siguiente texto de 1943: "Deogracia estaba muy pichona todavía, y a él francamente, no le gustaba nunca mirar los hombres arrochelados por allí, ojeándola" (Juan Pablo Sojo, Nochebuena negra, p. 15). De arrochela$d o^{2}$, este otro: "Dentro de los montes de Mata Oscura, Mata de Agua y Mata de Baúl estaba arrochelada gran cantidad de ganado" (Fernando Calzadilla Valdés, Por los llanos de Apure, p. 135; escrito entre 1927 y 1938), que, al parecer, no se corresponde tanto con la definición dada por ese diccionario de 'resistirse el ganado a seguir la marcha', como con la de 'estar reunido el ganado en algún lugar', acorde con una de las acepciones de rochela que ya tuvimos ocasión de comentar.

En cuanto al verbo, el DIVE respalda la primera acepción con un testimonio de 1772: “...en los parages donde concurren, se acogen o se arrochelan" (Archivo Nacional, Diversos, 6 de agosto, XLII, p. 411. [Decretos del Gobernador y Capitán General para matar los

11 Nótese, asimismo, que asigna la marca despectivo a la acepción 'aficionarse a un lugar', mas no a la $2^{\mathrm{a}}$ : "coloq Hacerse compañero frecuente de una persona”, acepción que equivale, a nuestro juicio, a 'aficionarse a una persona'. 
perros]). De la segunda acepción ('detenerse obstinadamente una bestia'), el DIVE afirma que está documentada en el Diccionario de barbarismos cotidianos de Juan Seijas, de 1890, y ofrece dos testimonios de principios del siglo Xx.

De los datos proporcionados por el CORDE y por el DIVE podemos entonces afirmar que los dos primeros testimonios de arrochelarse proceden -y esto es importante- de escritos llevados a cabo en territorio de la actual Venezuela fechados en el siglo XVIII, testimonios que traemos de nuevo aquí presentándolos conjuntamente:

ay en este valle vagamundos y algunos pícaros rrateros que se arrochelan en vn paraje que llaman Palma por no hauer Cabo ni ninguno que los persiga en el Valle al que acuden por la vecindad al Puerto: sus Naturales son desidiosos por lo común (1765, Juan de Salas, Ciudad de Nirgua. [Relaciones geográficas de la gobernación de Venezuela]).

...en los parages donde concurren, se acogen o se arrochelan (1772, Archivo Nacional, Diversos, 6 de agosto, XLII, p. 411. [Decretos del Gobernador y Capitán General para matar los perros]).

Ambos testimonios denotan la acción de reunirse y refugiarse, es decir, el verbo derivado conserva aquí dos de los significados de su raíz rochela; en el primero, refiriéndose a humanos malvivientes, en el segundo, a animales (en este caso, ya no al ganado, sino a perros).

Específicamente, de la forma arrochelado $-a$, no disponemos sino de testimonios relativamente tardíos. El primero, proporcionado por los corpus digitales, es el del texto colombiano ya visto, que, pese a estar fechado en 1992, remite a una situación de la época colonial (s. XVIII), pero de la que desconocemos el año preciso: "evitar que las gentes se establecieran «arrocheladas», como se decía a la colonización espontánea”. Establecerse en un lugar, aunque sea de manera desordenada, espontánea, daría pie ciertamente a que esas personas se aquerenciaran en él, en un sentido lato del término. Entre 'establecerse en un lugar' y 'frecuentarlo' hay una evidente relación semántica que explicaría el empleo de arrochelado en distintas situaciones que tendrían en común el rasgo 'estar'. Del significado que la voz tiene en nuestro legajo merideño, el DIVE presenta como testimonio más antiguo -ya lo hemos visto- el siguiente texto de 1943: "Deogracia estaba muy pichona todavía, y a él francamente, no le gustaba nunca mirar los hombres arrochelados por allí, ojeándola" (Juan Pablo Sojo, Nochebuena negra, p. 15). Esos hombres enamoradizos que van y vienen a menudo por las calles y esquinas cercanas a la casa donde viven muchachas objeto de sus deseos están, sin embargo, representados siglo y medio antes en nuestro Francisco Ramírez, el merideño acusado de haber seducido a Rafaela. En consecuencia, es el legajo sobre estupro el que, hasta prueba de lo contrario, se constituye en 
primer testimonio (1786) del uso de arrochelado referido a "persona que frecuenta regularmente un sitio, particularmente por intereses amorosos'.

El orden cronológico de las diversas acepciones de arrochelarse (y de su participio pasado arrochelado) sería, entonces, según lo que hemos podido averiguar con la documentación disponible hasta ahora, el siguiente: 'refugiarse fugitivos y malvivientes' (1765), 'habituarse un animal a un sitio' (1772), 'frecuentar una persona un determinado lugar' (1786), 'plantarse la caballería' (1917). El año entre paréntesis es el del texto más antiguo proporcionado por las fuentes consultadas, y en ningún caso puede tomarse como primer testimonio sino de manera relativa y provisional. Lo único seguro es que, excepto quizá la última, todas las acepciones ya estaban en uso en Venezuela hacia mediados de la $18^{\mathrm{a}}$ centuria.

El glosario que recoge, define y ejemplifica de manera magistral la palabra en cuestión, sintetizando prácticamente todas las acepciones que ha tenido y tiene en Venezuela, es el Léxico popular venezolano (Tamayo 1977), al afirmar que arrochelado

Se dice de aquella persona o animal que se estaciona frecuentemente en un sitio dado, el cual es distinto del que les es propio o les había sido habitual. Ej.: "Juan está arrochelado en la esquina de las Pérez; parece que está enamorado por allí". "El ganado está arrochelado en el estero".

No hemos querido terminar esta nota sin antes averiguar cuál es la situación de arrochelarse y arrochelado en el español hodierno de Venezuela. Si nos atenemos a la información proporcionada por el CREA y por el CORPES XXI de la RAE ${ }^{12}$, información siempre limitada, incompleta y provisional, como la de todo corpus, dichas palabras serían hoy por hoy muy poco usuales en este país. El CREA no da ningún ejemplo venezolano; únicamente trae el del texto colombiano de 1992 ya comentado. El CORPES XXI exhibe apenas 4 ejemplos, todos ellos venezolanos, tomados de una misma obra (Veinte cuentos malucos, Caracas, 2001) de Lorenzo Álvarez, ejemplos que se transcriben a continuación:

1. Tampoco faltaban los espantos que se arrochelaban hasta llevarse a alguien como bastimento.

2. Media familia Renterías se arrocheló en la vivienda de los Sulbarán... 3. La carcacha dio muestras de tener necedades propias cuando viajaba hacia Paso Fino, y cada vez que transitaba por la subida de El Burro ubicada frente a la posada de Los Capinos se arrochelaba y allí se quedaba como muerta.

12 Recuérdese que el CREA se compone de textos escritos y orales realizados en el mundo hispanohablante entre 1975 y 2004. El CORPES XXI, en cambio, va de 2001 a 2016. Sobre ambos corpus, véase www.rae.es. 
4. No hubo preámbulo para serenatas; al siguiente día ya estaban casados y arrochelados en una hamaca...

En los ejemplos 1 y 2, arrochelarse equivale a 'juntarse, reunirse en un lugar distinto al propio o habitual'; en el 4, a ese sentido parece agregarse el de 'enamoramiento', que tiene, como ya se vio, la raíz rochela; en el ejemplo 3, el autor amplía la acepción de 'plantarse la caballería' para referirse a un vehículo en malas condiciones que se accidentaba en el sitio mencionado y no podía (¿quería?) seguir andando. Se conservan, como se ve, acepciones ya conocidas. Los muy pocos ejemplos encontrados pudieran hacer pensar, pues, que el empleo de arrochelarse y su participio es actualmente escaso en Venezuela, pero esta conjetura sólo podrá verificarse después de análisis más detenidos y con corpus de mayor extensión que demuestren cuán vivo o no está el uso de las voces objeto de este trabajo.

\section{A MODO DE CONCLUSIÓN}

Hasta aquí llega nuestro análisis de arrochelarse y su participio arrochelado. De estas formas léxicas y de su raíz rochela podemos concluir, tomando en cuenta todo lo expuesto, que son -desde una perspectiva únicamente sincrónica-venezolanismos. Arrochelado lo sería en un grado superior (si de grados puede hablarse) por sus significados, uso y extensión ${ }^{13}$; en otras palabras, por ser una voz empleada en el español general de Venezuela inexistente en otras variedades del diasistema español (si nos atenemos a lo que señalan las fuentes). En el caso de rochela, hablaríamos de un venezolanismo semántico por haber desarrollado en este país valores que le son propios, no usuales en otras variedades de la lengua, aunque comparta con Colombia una de sus acepciones ('bullicio, algazara'), como ya se vio en su momento. Desde la perspectiva diacrónica, rochela tiene su origen en el español peninsular con su primitivo valor de 'lugar de confusión, desorden, pillaje' ${ }^{\prime 4}$, significado que tendría también en parte de América y sobre el cual habrían de desarrollarse luego en este continente, y particularmente en Venezuela, otras acepciones. Coincidimos con Ramírez Luengo (2014) cuando afirma que adscribir determinadas unidades léxicas a alguno de los tres tipos de -ismos propuestos por Company (2010) (puros, de frecuencia y semánticos), puede ser problemático "-muy especialmente para otros momentos de la historia- para poder clasificarlos según sus propias especificidades” (p. 3), porque, glosando a dicho investigador, no todos los vocablos resultan propios

13 Asimilable quizás a lo que Company (2005 y 2010) llama -ismos puros.

14 Véase supra, nota 7. 
de algún lugar de la misma manera, lo que se ha hecho patente en el caso de arrochelarse, arrochelado y rochela.

\section{REFERENCIAS}

\section{Fuentes documentales}

Causa de Xavier de Lobo contra Francisco Ramírez por haberle éste seducido y gravidado una hija menor 1786. Archivo General del Estado Mérida (AGEM), Estupro, Tomo 1, ff. $5 \mathrm{r}-15 \mathrm{r}$.

\section{Bancos de datos}

$\mathrm{CDH}=$ Instituto de Investigación Rafael Lapesa de la Real Academia Española 2013. Corpus del Nuevo diccionario histórico $(C D H)$ [en línea], http:/ / web.frl.es/CNDHE [consultado el 11 de abril de 2018].

CORDE = Real Academia Española: Banco de datos (CORDE) [en línea]. Corpus diacrónico del español, http://www.rae.es [consultado el 9 de abril de 2018].

CORDIAM = Academia Mexicana de la Lengua, Corpus diacrónico y diatópico del español de América (CORDIAM), www.cordiam.org [consultado el 12 de abril de 2018].

CORPES XXI = Real Academia Española: Banco de datos (CORPES XXI) [en línea]. Corpus del español del Siglo XXI (CORPES), http://www.rae.es [consultado el 17 de abril de 2018].

CREA = Real Academia Española: Banco de datos (CREA) [en línea]. Corpus de referencia del español actual, http://www.rae.es [consultado el 14 de abril de 2018].

\section{Repertorios lexicográficos}

Alemany y Bolufer, José 1917. Diccionario de la lengua española, http:/ / www.rae.es / recursos/diccionarios/diccionarios-anteriores-1726-1992/nuevo-tesoro-lexicografico [consultado el 10 abril de 2018].

Asociación de Academias de la Lengua Española (ASALE) 2010. Diccionario de americanismos (DAMER), http:/ /lema.rae.es/damer/ [consultado el 13 abril de 2018].

Corominas, Joan y José Antonio Pascual 1980-1991. Diccionario crítico etimológico castellano e hispánico, Gredos, Madrid, 6 ts.

Núñez, Rocío y Francisco Javier Pérez 1994. Diccionario del habla actual de Venezuela (DHAV), Universidad Católica Andrés Bello, Caracas.

Real Academia Española 1927. Diccionario de la lengua española (DRAE), http://www. rae.es / recursos / diccionarios / diccionarios-anteriores-1726-1992/ nuevo-tesoro-lexicografico [consultado el 10 abril de 2018].

Real Academia Española 1985. Diccionario de la lengua española (DRAE), http://www. rae.es / recursos / diccionarios / diccionarios-anteriores-1726-1992/ nuevo-tesoro-lexicografico [consultado el 10 abril de 2018].

Real Academia Española 2014. Diccionario de la lengua española (DLE), http:/ dle.rae. es / ?w=diccionario [consultado el 12 de abril de 2018].

Real Academia Española s.f. Mapa de diccionarios académicos, http://www.rae.es/ recursos / diccionarios/ diccionarios-anteriores-1726-1992/mapa-de-diccionarios [consultado el 12 de abril de 2018]. 
Tamayo, Francisco 1977. Léxico popular venezolano, Universidad Central de Venezuela, Caracas.

Tejera, María Josefina (dir.) 1993. Diccionario de venezolanismos (DIVE), Universidad Central de Venezuela-Academia Venezolana de la Lengua, Caracas.

Zerolo, Elías et al. 1895. Diccionario enciclopédico de la lengua castellana, http:/ /www. rae.es/ recursos/ diccionarios/ diccionarios-anteriores-1726-1992/ nuevo-tesoro-lexicografico [consultado el 10 de abril de 2018].

\section{Referencias bibliográficas}

Company Company, Concepción 2005. "El siglo XviII y la identidad lingüística de México". Discurso de ingreso a la Academia Mexicana de la Lengua, http://www. academia.org.mx/ sesiones-publicas/ item/ ceremonia-de-ingreso-de-dona-concepcion-company-company [consultado el 5 de mayo de 2018].

Company Company, Concepción 2010. "Introducción", en Academia Mexicana de la Lengua, Diccionario de mexicanismos, pp. xv-xxiii.

Medina Ceballos, Jílmer Johán 2014. Selección, transcripción paleográfica y análisis lingüistico de manuscritos fechados en los siglos XVIII y XIX, conservados en el Archivo General de la Nación (AGN), informe de pasantía, Universidad Central de Venezuela, Caracas.

Ramírez Luengo, José Luis 2014 “Cómo el español de España genera americanismos: a propósito del americanismo puto 'homosexual”, Lengua y Habla, 18, pp. $1-12$.

Rosenblat, Ángel 1974. Buenas y malas palabras, Edime, Madrid, 4 ts. 
